\title{
Leukemia Candidate Genetic Markers-An Evaluation
}

\author{
Xixi Xiang ${ }^{1}$, Parker Foster ${ }^{2}$, Xi Zhang ${ }^{1}$, Xiangning $\mathrm{Bu}^{3^{*}}$ \\ ${ }^{1}$ Department of Hematology, Xinqiao Hospital, Third Military Medical University, \\ Xinqiao, Chongqing 400037, P.R. China; \\ ${ }^{2}$ National Institute of Biomedical Imaging and Bioengineering (NIBIB), NIH, \\ Bethesda, MD 20892, USA; \\ ${ }^{3}$ National Heart, Lung, and Blood Institute (NHLBI), NIH, Bethesda, MD 20814 , \\ USA.
}

*Corresponding Author: Xiangning Bu, Ph.D. National Heart, Lung, and Blood Institute (NHLBI), NIH, Bethesda, MD, 20852, USA. Tel: +1 (301) 594-7359; Email: xiangning.bu@nih.gov.

\section{ABSTRACT}

Background: The cause of leukemia, the most common type of cancer, remains unknown. Genetic studies have reported more than a thousand of genes as being linked to the disease.

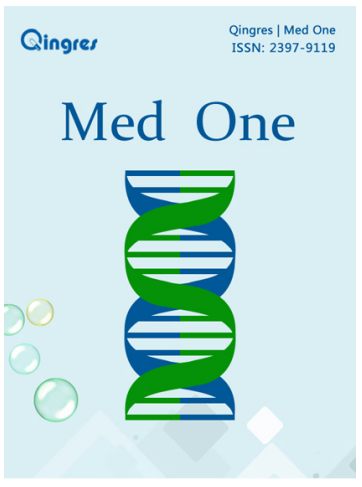

http://mo.qingres.com

GOPEN ACCESS

DOI: 10.20900/mo.20160019

Received: May 24, 2016

Accepted: July 18, 2016

Published: August 25, 2016

Copyright: ๑2016 Cain et al. This is an open access article distributed under the terms of the Creative Commons Attribution License, which permits unrestricted use, distribution, and reproduction in any medium, provided the original author and source are credited.
Methods: A total of 1,093 leukemia candidate genes, identified from leukemia-gene relations data extracted from the ResNet 11 Mammalian database and supported by 6,524 references were evaluated. Four network metrics were used to evaluate individual gene potential relevance to leukemia. Gene-set enrichment, sub-network enrichment, and network-connectivity analyses were conducted on gene attributes. An expression dataset of 71 leukemia patients, and 76 healthy controls, was employed for validation.

Results: A total of 952 out of 1,093 genes were enriched in 100 pathways $(p<3.3 \mathrm{e}-20)$, demonstrating strong gene-gene interaction. A network metrics analysis revealed 5 genes (TP53, CTNNB1, AKT1, TNF, and RARA), as measured by both functional diversity and replication frequency, as the top leukemia candidates. Validation, using expression data, showed that the 1,093 genes, as a whole, and the top genes, as identified by the proposed metrics, were efficient in distinguishing leukemia patients from controls (maximum classification ratio $=95.3 \%$ with permutation $p$-value $=0.0054)$.

Conclusion: The genetic causes of leukemia are linked to a genetic network composed of a large number of genes. This network, together with the network metrics provided in this study, could provide a basis for further molecular studies in the field.

Key Words: Leukemia; ResNet Database; Gene set enrichment 
analysis; Sub-network enrichment analysis; Network connectivity analysis; LOO cross validation

\section{INTRODUCTION}

Leukemia is a group of cancers, usually originating in bone marrow, which result in great numbers of abnormal white blood cells. It is the most common type of childhood cancer, even though approximately $90 \%$ of all leukemia cases are in adults ${ }^{[1]}$. The precise causes of leukemia remain unknown. Inherited and environmental factors are both thought to be involved ${ }^{[2]}$.

More than a thousand genes related to leukemia, many of which suggested as potential biomarkers for the disease, such as FLT3, WT1, TET2, and KRAS, have been reported ${ }^{[3-5]}$. Several genes, such as IL2 and CSF3, have been studied in clinical trials ${ }^{[6,7]}$. Many articles have reported genetic changes, and gene quantitative changes, in leukemia ${ }^{[8,9]}$. Both increased, and decreased, gene expression levels/activities have been observed ${ }^{[10-}$ ${ }^{12]}$. Many genes have been reported to influence leukemia pathogenic development via unknown mechanisms $^{[13]}$.

We found no study reporting a systematic evaluation of the quality, and strength, of these reported genes as a functional network/group in the underlying biological process of leukemia. This study, instead of focusing on specific genes, attempts to provide a comprehensive view of the genetic-map, and use gene set enrichment analysis (GSEA) and sub-network enrichment analysis (SNEA) to study the underlying functional profiles of the genes identified $^{[14]}$. The hypothesis is that leukemia genes are functionally linked to each other and co-regulate leukemia's pathogenic development via multiple pathways.

\section{MATERIALS AND METHODS}

The study workflow was as follows: 1) acquisition of a leukemia-gene relation dataset and identification of leukemia candidate genes; 2) enrichment analysis of the identified genes to study their pathogenic significance to leukemia; 3) network metrics analysis to identify genes having specific significance; 4) network connectivity analysis (NCA) to test functional associations between the reported genes; and, 5) validation using an independent gene expression data set.

\subsection{Leukemia-Gene relation Data Acquisition}

Leukemia-gene relation data were extracted from the Pathway Studio ResNet@ Mammalian database updated as of May 2016. The genes identified were used as the candidate network nodes/genes.

The ResNet $®$ Mammalian database was a part of the Pathway Studio ResNet Databases. This is a group of real-time updated network databases and includes: curated signaling; cellular process and metabolic pathways; ontologies and annotations; and, molecular interactions and functional relationships extracted from the $35 \mathrm{M}+$ references covering the entire PubMed abstract and Elsevier full text journals. It is updated weekly. The ResNet ${ }^{\circ}$ Mammalian database contains information for more than 6,500,000 functional relationships for humans, rats, and mice and is linked to all of the original literature sources. The database includes: 1) 142,270 proteins; 2) 106,732 small molecules; 3 ) 8,863 cell processes; 4) 15,911 diseases; 5) 5,038 functional classes; 6) 4,387 Clinical parameters; 7) 1,983 pathways; 8) 559 complexes; and, 9) 767 cells. (ResNet databases, http: //pathwaystudio. gousinfo.com/ResNetDatabase.html) .

\subsection{Literature metrics analysis}

There were 2 scores proposed for each genedisease relationship as a literature metrics analysis.

The reference number underlying a gene-disease relationship as the gene reference score (RScore) is defined by Eq.(1).

RScore $=$ The number of references underlying a relationship

The earliest publication age of a gene-disease relationship is the gene age score (AScore) and is defined by Eq.(2)

$$
\text { AScore }=\max _{1 \leq i \leq n} \text { ArticlePubAge }_{i}
$$

where $n$ is the total number of references supporting a gene-disease relation, and

ArticlePubAge $=$ Current date-Publication date +1

\subsection{Enrichment metric analysis}

Given a disease associated with a set of genetic 
pathways $\mathscr{R}$ the gene-wise enrichment score (EScore) for the kth gene, within a gene set of size $n$, is defined in Eq. (4) as

$$
\text { EScore }_{k}=\sum_{i-1}^{m}\left(-\log _{10} p \text { Value }_{i}\right) / \max _{1<i<n}\left(-\log _{10} \mathrm{pValue}_{\mathrm{i}}\right)
$$

where $p$ Value $_{i}$ is the enrichment score of the ith pathway with the gene set; $m \in \mathscr{R}$ is the number of pathways including kth the gene. The PScore for the gene, $\mathrm{m}$, is defined as

PScorek $=$ The number of pathways that form $\mathscr{R}$ including the $k$ th gene

The PScore presents how many disease-related pathways were associated with the genes. The EScore shows involved pathway significance.

\subsection{Enrichment analysis}

GSEA and SNEA ${ }^{[15]}$ were performed on: 1) entire gene list (1,093 genes); and 2) 2-subgroups with the highest metric scores to better understand any underlying functional profiles and gene pathogenic significance. An NCA was conducted on the two 2-subgroups.

\subsection{Validation using gene expression data}

The hypothesis is that significant leukemia candidate gene-gene sets should be a factor in distinguishing leukemia patients from healthy controls. A Euclidean distance-based multivariate classification ${ }^{[16]}$ on an expression dataset, followed by a leave-oneout (LOO) cross validation, using the overall gene set and the sub-sets selected by different scores as tentative markers was performed to evaluate the effectiveness of the selected genes and the proposed metrics

A permutation of 5,000 runs was then conducted to test the hypothesis that a randomly selected gene set of the same size could lead to equal, or better, classification accuracy.

Expression data from 147 subjects, including samples from 71 chronic, lymphocytic leukemia (CLL) tumors, and 76 sorted CD19pos B cells from healthy donors (NCBI GEO: GSE50006), with 1,031 genes overlapped with the candidate leukemia gene-pool identified within the leukemia-gene dataset.

\section{RESULTS}

\subsection{Identification of candidate genes}

There were 1,093 leukemia candidate genes identified from the leukemia-gene relation data set. They are supported by 6,524 articles (Supplementary Material 1).There were 994 (90.94\%) which presented a regulation relationship to the disease; 133 (12.17\%) a genetic change; 61 (5.58\%) a quantitative change; $52(4.76 \%)$ cell expression; 20 $(1.83 \%)$ with Biomarker, $17(1.56 \%)$ with clinical trial, and $5(0.46 \%)$ with state changes. There are $148(13.54 \%)$ genes that have been reported to have multiple relationships with the disease. There were 945 (86.46 \%) genes that presented a 1-type relationship to the disease, $113(10.34 \%)$ with a 2 , $31(2.84 \%)$ with a $3,3(0.27 \%)$ with a 4 , and $1(0.09$ $\%$ ) with a 5 . For a detailed definition and description of these relation types mentioned above, refer to the 'Relations: Definitions and Annotations' section at http://pathwaystudio. Gousinfo. com/ ResNet Database. html. Genes with ' $m$ ' and ' $r$ ' are genes identified in mice and rats, respectively.

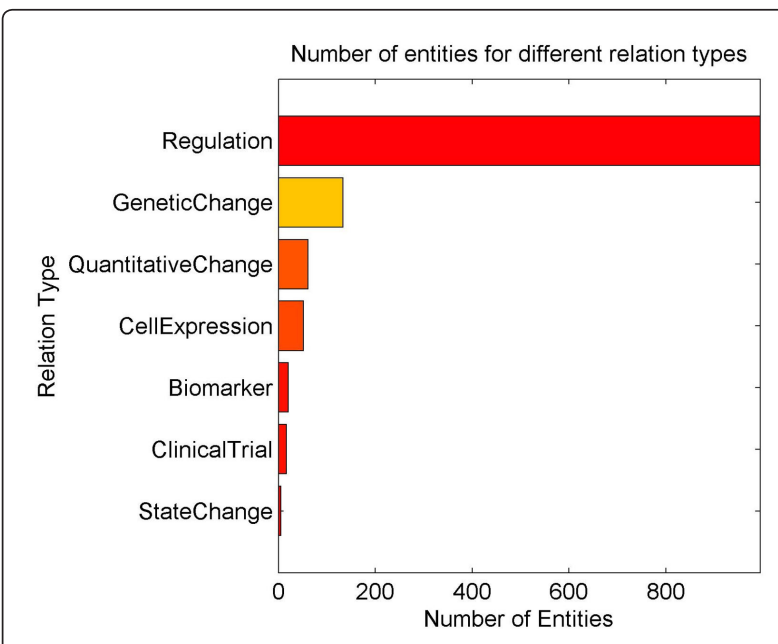

Fig. 1 Gene Relation Type Distribution of the 1,093 Genes

The publication date distribution for these 6,531 articles appears in Fig. 2 (a). Novel genes are reported in each year. These have an average publication age of 6.0 years indicating that most were published recently. Publication date distributions for most of the articles underlying the 1,093 genes were similar (Fig. 2 (b)). 


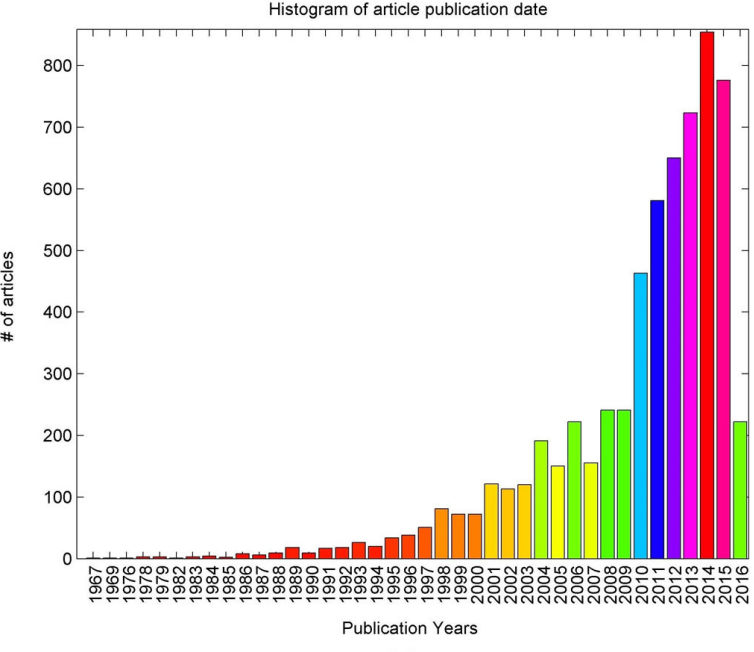

(a)

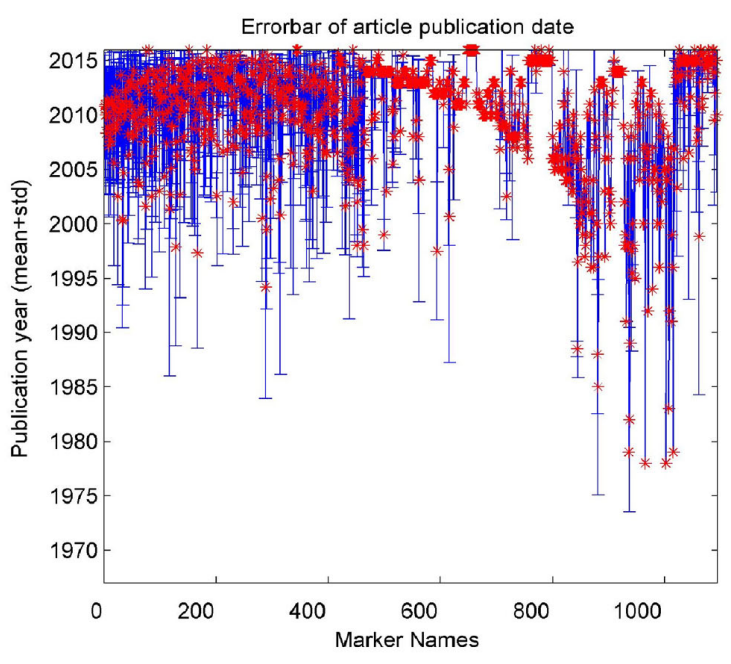

(b)

Fig. 2 Histogram of publications reporting gene-disease relationships between leukemia and the 1,093 Genes. (a) Number of articles published by year; (b) Gene-wise publication date distribution of the supporting references, with mean marked as red star.

\subsection{Marker ranking}

Of these 1,093 genes, 31 were reported in the period January through April within this year 2016 (Table 1).
Table 1 also lists the top 31 genes with the highest RScores (in descending order). Full results appear in Supplementary Material 1.

Table 1. Top 31 Genes with Reported Associations to Leukemia Ranked by Different Scores

$\begin{array}{ll} & \text { SPN; HHEX; RING1; ESAM; ATMIN; KDM4C; RNF2; ABCC4; BMP15; CDKN 1C; } \\ \text { Genes with } & \text { CDKN2C; DVL1; DVL3; DYNLL1; HDAC3; IDDM2; ITK; LDB1; P2RX2; P 2RY14; } \\ \text { Ascore=1 } & \text { PCBP2; PFKFB3; PRDX2; PRD X4; RHOXF2; SHCBP1; SMARCA2; TES; TJP1; } \\ & \text { TNFSF11; TRPV2 } \\ & \text { KMT2A; NOTCH1; NPM1; PTPN11; PTEN; HOXA9; BCL2; WT1; MEIS1; CSF2 ; } \\ \text { Genes by } & \text { DNMT3A; IDH1; IDH2; FLT3; TP53; M YC; ABL1; CTNNB1; CSF3; SPI1; IL2; A KT1; } \\ \text { Rscore } & \text { TAL1; TNF; VEGFA; RARA; RUNX1; MECOM; TET2; KIT; ASXL1 }\end{array}$

\subsection{Enrichment analysis}

This section presents GSEA and SNEA results for 3 different groups: the 1,093 genes, and both gene groups listed in Table 1.

\subsubsection{Enrichment analysis of the 1,093 genes}

Table 2 presents the top 20 pathways/groups enriched with $857 / 1,093$ genes ( $p$-values $<3.7$ e41). A complete list of the 100 pathways/gene sets enriched with 952/1,093 genes ( $p$-value $<3.3 \mathrm{e}-20)$ appears in Supplementary Material 2.

Among the 100 pathway/groups enriched, 6 related to cell apoptosis (345/1,093 genes), 9 to cell growth and proliferation (366/1,093 genes), 6 to protein phosphorylation (201/1,093 genes), 3 to the immune system (319/1,093 genes), 11 to transcription factors (449/1,093 genes), 8 to protein kinase (234/1,093 genes), and 2 to neuronal systems (257/1,093 genes). 
Table 2. Molecular Function Pathways/Groups Enriched by 1,093 Genes Reported

\begin{tabular}{|c|c|c|c|c|c|c|}
\hline Pathway/gene set name & Hit type & GO ID & $\begin{array}{c}\text { \#of } \\
\text { Entities }\end{array}$ & Overlap & $p$-value & $\begin{array}{l}\text { Jaccard } \\
\text { similarity }\end{array}$ \\
\hline $\begin{array}{l}\text { Positive regulation of } \\
\text { transcription from RNA } \\
\text { polymerase II promoter }\end{array}$ & Biological process & 0010552 & 1041 & 228 & $1.22 \mathrm{E}-91$ & 0.12 \\
\hline $\begin{array}{l}\text { Positive regulation of cell } \\
\text { proliferation }\end{array}$ & Biological process & 0008284 & 568 & 163 & 4.35E-83 & 0.11 \\
\hline $\begin{array}{l}\text { Negative regulation of } \\
\text { apoptotic process }\end{array}$ & Biological process & 0006916 & 650 & 170 & 7.97E-80 & 0.11 \\
\hline Response to drug & Biological process & 0017035 & 509 & 138 & 1.57E-66 & 0.09 \\
\hline $\begin{array}{l}\text { Positive regulation of } \\
\text { transcription, DNA- } \\
\text { templated }\end{array}$ & Biological process & 0045941 & 623 & 149 & $5.84 \mathrm{E}-64$ & 0.1 \\
\hline Cytosol & Cellular component & 0005829 & 3173 & 353 & 2.36E-63 & 0.09 \\
\hline Nucleoplasm & Cellular component & 0005654 & 2669 & 317 & 2.16E-62 & 0.09 \\
\hline Innate immune response & Biological process & 0002226 & 792 & 159 & $4.50 \mathrm{E}-57$ & 0.09 \\
\hline $\begin{array}{l}\text { Negative regulation of } \\
\text { Transcription from RNA } \\
\text { polymerase II promoter }\end{array}$ & Biological process & 0000122 & 799 & 154 & 9.07E-53 & 0.09 \\
\hline $\begin{array}{l}\text { Neurotrophin TRK } \\
\text { receptor signaling } \\
\text { pathway }\end{array}$ & Biological process & 0048011 & 280 & 91 & $3.66 \mathrm{E}-51$ & 0.07 \\
\hline $\begin{array}{l}\text { Transcription, DNA- } \\
\text { templated }\end{array}$ & Biological process & 0061018 & 3130 & 280 & $1.56 \mathrm{E}-48$ & 0.09 \\
\hline Apoptotic process & Biological process & 0008632 & 790 & 145 & $6.95 \mathrm{E}-47$ & 0.08 \\
\hline $\begin{array}{l}\text { Response to organic } \\
\text { cyclic compound }\end{array}$ & Biological process & 0014070 & 253 & 82 & 4.87E-46 & 0.07 \\
\hline $\begin{array}{l}\text { Regulation of } \\
\text { transcription, DNA- } \\
\text { templated }\end{array}$ & Biological process & 0061019 & 2670 & 291 & $1.02 E-45$ & 0.08 \\
\hline Blood coagulation & Biological process & 0007596 & 501 & 112 & $9.89 E-45$ & 0.08 \\
\hline $\begin{array}{l}\text { Positive regulation of } \\
\text { apoptotic process }\end{array}$ & Biological process & 0043065 & 393 & 98 & $1.41 \mathrm{E}-43$ & 0.07 \\
\hline $\begin{array}{l}\text { Fc-epsilon receptor } \\
\text { signaling pathway }\end{array}$ & Biological process & 0038095 & 186 & 68 & $3.48 \mathrm{E}-42$ & 0.06 \\
\hline $\begin{array}{l}\text { Response to } \\
\text { lipopolysaccharide }\end{array}$ & Biological process & 0033196 & 252 & 78 & $3.60 \mathrm{E}-42$ & 0.06 \\
\hline $\begin{array}{l}\text { Negative cell proliferation } \\
\text { regulation }\end{array}$ & Biological process & 0008285 & 471 & 105 & $8.20 \mathrm{E}-42$ & 0.07 \\
\hline $\begin{array}{l}\text { Transcription factor } \\
\text { binding }\end{array}$ & Molecular function & 0008134 & 326 & 89 & $3.66 \mathrm{E}-41$ & 0.07 \\
\hline
\end{tabular}

Note: A Jaccard similarity is a statistic used to compare the similarity and diversity of two sample sets, which is defined by , where $A$ and $B$ are two sample sets. 
The top 10 disease-related sub-networks enriched with a $p$-value < 5E-254 appear in Table 3. Complete results appear in Supplementary Material 3.

Table 3. Sub-networks Enriched by the 1,093 Genes

\begin{tabular}{|c|c|c|c|c|}
\hline Gene Set Seed & Total \# of Neighbors & Overlap & $p$-value & $\begin{array}{l}\text { Jaccard } \\
\text { Similarity }\end{array}$ \\
\hline Breast Cancer & 3114 & 641 & $<1 \mathrm{E}-324$ & 0.18 \\
\hline Leukemia, Myeloid, Acute & 1008 & 498 & $<1 \mathrm{E}-324$ & 0.32 \\
\hline Lymphoma & 952 & 408 & $<1 \mathrm{E}-324$ & 0.25 \\
\hline Carcinogenesis & 1686 & 482 & 3.2E-287 & 0.21 \\
\hline $\begin{array}{l}\text { Precursor Cell Lymphoblastic } \\
\text { Leukemia-Lymphoma }\end{array}$ & 577 & 309 & $1.9 \mathrm{E}-273$ & 0.23 \\
\hline Lung Cancer & 1708 & 465 & $1.2 \mathrm{E}-264$ & 0.2 \\
\hline Carcinoma, Hepatocellular & 2395 & 534 & 4.3E-264 & 0.18 \\
\hline Carcinoma, Non-Small-Cell Lung & 1517 & 441 & 4.1E-262 & 0.21 \\
\hline Colorectal Cancer & 2261 & 517 & $2 E-259$ & 0.18 \\
\hline Prostate Cancer & 1937 & 480 & 4.1E-254 & 0.19 \\
\hline
\end{tabular}

Many of these reported leukemia-related genes are associated with other cancers that were linked to Leukemia, with a large overlap (Jaccard similarity $>0.18$ ).

\subsubsection{Enrichment analysis on top $\mathbf{3 1}$ genes with highest scores}

The GSEA and SNEA results of the top 31 genes listed in Table 1 were compared. The top 10 pathways/sub-networks for the AScore group and the RScore group are presented (Table 4 and Table 5). Complete results appear in Supplementary Material 2 and 3.
Using a $p$-value threshold $(p<1 \mathrm{E}-4)$, the 31 genes with top AScores were enriched within 10 pathways/ groups. The RScore group score was 153.

The top 10 pathways enriched with the 31 genes from the AScore and RScore groups appear in Table 4. A complete listing of these pathways/gene sets appears in Supplementary Material 2. 
Table 4. Pathways/Groups Enriched by 31 Genes with the Highest AScore and RScore

\begin{tabular}{|c|c|c|c|}
\hline & Pathway/gene set Name & GO ID & $p$-value \\
\hline \multirow{10}{*}{$\begin{array}{l}\text { The first } 10 \\
\text { pathways/gene } \\
\text { sets enriched by } \\
\text { top } 31 \text { genes with } \\
\text { highest AScore }\end{array}$} & Dvl & Pathway Studio Ontology & 7.62E-07 \\
\hline & AhpC/TSA family & Pathway Studio Ontology & $1.52 \mathrm{E}-06$ \\
\hline & $\begin{array}{l}\text { Negative regulation of } \\
\text { phosphorylation }\end{array}$ & 0042326 & $5.23 \mathrm{E}-06$ \\
\hline & Peroxiredoxin & Pathway Studio Ontology & 7.10E-06 \\
\hline & Chromatin binding & 0003682 & $8.48 \mathrm{E}-06$ \\
\hline & Enzyme binding & 0019899 & 1.63E-05 \\
\hline & Thioredoxin peroxidase activity & 0008379 & 2.22E-05 \\
\hline & Peroxiredoxin activity & 0051920 & 4.65E-05 \\
\hline & Sex chromatin & 0001739 & 4.73E-05 \\
\hline & $\begin{array}{l}\text { Positive regulation of protein } \\
\text { Phosphorylation }\end{array}$ & 0001934 & $9.23 \mathrm{E}-05$ \\
\hline \multirow{10}{*}{$\begin{array}{l}\text { The first } 10 \\
\text { pathways/gene } \\
\text { sets enriched by } \\
\text { top } 31 \text { genes with } \\
\text { highest RScore }\end{array}$} & $\begin{array}{l}\text { Positive regulation of transcription } \\
\text { from RNA Polymerase II promoter }\end{array}$ & 0010552 & $1.12 \mathrm{E}-17$ \\
\hline & $\begin{array}{l}\text { Positive regulation of cell } \\
\text { proliferation }\end{array}$ & 0008284 & $1.68 \mathrm{E}-14$ \\
\hline & $\begin{array}{l}\text { Negative regulation of cell } \\
\text { proliferation }\end{array}$ & 0008285 & 1.36E-12 \\
\hline & $\begin{array}{l}\text { Positive regulation of transcription, } \\
\text { DNA-templated }\end{array}$ & 0045941 & $3.55 \mathrm{E}-11$ \\
\hline & Regulation of cell proliferation & 0042127 & 4.66E-11 \\
\hline & Oncogenes & Pathway Studio Ontology & $4.71 \mathrm{E}-11$ \\
\hline & $\begin{array}{l}\text { Lymphoid progenitor cell } \\
\text { differentiation }\end{array}$ & 0002320 & $1.36 \mathrm{E}-10$ \\
\hline & Hemopoiesis & 0030097 & $1.53 \mathrm{E}-10$ \\
\hline & Enzyme binding & 0019899 & $1.98 \mathrm{E}-10$ \\
\hline & $\begin{array}{l}\text { Myeloid progenitor cell } \\
\text { differentiation }\end{array}$ & 0002318 & $2.71 \mathrm{E}-10$ \\
\hline
\end{tabular}


Genes with the top AScores and those with the top RScores enriched different groups of pathways with different $p$-values (AScore group: 7.62E-07 - 9.23E-05; RScore group: 1.12E-17 - 2.71E-10). This suggests that the newly-reported genes are both functionally distinct, and are less significant, compared to those most frequently reported.

It was observed that 4/10 pathways/gene sets enriched by the RScore group (Table 4) in Table 2 also appear in the top 20 pathways/groups enriched with 857/1,093 genes. The AScore group had none.

Results from the SNEA analysis consist of an enrichment analysis against disease sub-networks. Table 5 presents the top 10 disease-related subnetworks enriched by the top 31 genes from the AScore group and the RScore group, respectively. Complete results appear in Supplementary Material 3.

Table 5. SNEA Results by 31 Genes with the Highest AScore and RScore

\begin{tabular}{|c|c|c|c|c|}
\hline & Gene Set Seed & Overlap & $p$-value & $\begin{array}{l}\text { Jaccard } \\
\text { similarity }\end{array}$ \\
\hline \multirow{10}{*}{$\begin{array}{l}\text { The first } 10 \\
\text { pathways/gene } \\
\text { sets enriched } \\
\text { by top } 31\end{array}$} & Carcinoma, Pancreatic Ductal & 6 & 4.90E-05 & 0.01 \\
\hline & Cystitis, Interstitial & 3 & 5.19E-05 & 0.03 \\
\hline & Primary tumor & 7 & 8.84E-05 & 0.01 \\
\hline & Lymphoma & 7 & 9.96E-05 & 0.01 \\
\hline & Breast Cancer & 12 & 1.19E-04 & 0 \\
\hline & Melanoma & 8 & 1.43E-04 & 0.01 \\
\hline & Carcinoma & 8 & $1.95 E-04$ & 0.01 \\
\hline & Extravasation & 3 & 2.39E-04 & 0.02 \\
\hline & Diabetes Mellitus & 10 & 2.65E-04 & 0 \\
\hline & Anemia & 5 & $2.72 \mathrm{E}-04$ & 0.01 \\
\hline \multirow{10}{*}{$\begin{array}{l}\text { The first } 10 \text { gene } \\
\text { sets Enriched } \\
\text { highest RScore }\end{array}$} & Leukemia, Myeloid & 27 & $1.41 \mathrm{E}-52$ & 0.12 \\
\hline & $\begin{array}{l}\text { Leukemia, Myelogenous, } \\
\text { Chronic, BCR-ABL Positive }\end{array}$ & 30 & $1.85 E-52$ & 0.07 \\
\hline & Leukemogenesis & 26 & $1.99 E-50$ & 0.13 \\
\hline & Acute leukemia & 27 & 1.13E-46 & 0.08 \\
\hline & Myeloproliferative Disorders & 24 & $2.05 E-45$ & 0.12 \\
\hline & Neoplasm, Residual & 22 & $6.24 \mathrm{E}-45$ & 0.17 \\
\hline & Myelodysplastic Syndromes & 28 & $8.90 \mathrm{E}-45$ & 0.06 \\
\hline & Blast Crisis & 22 & $8.44 \mathrm{E}-44$ & 0.15 \\
\hline & Leukemia, Myeloid, Acute & 31 & $8.80 \mathrm{E}-44$ & 0.03 \\
\hline & Leukemia, Promyelocytic, Acute & 23 & $9.32 \mathrm{E}-41$ & 0.1 \\
\hline
\end{tabular}

From Table 5, both groups enriched other cancer related sub-networks. Enrichment p-values of the RScore group were much more significant than those of the AScore group (NScore group: 4.90E-05 - 2.72E-04; RScore group: $1.41 \mathrm{E}-52$ - 9.32E-41), and have greater Jaccard similarities. 


\subsection{Connectivity analysis}

An NCA was performed on the top 31 genes with the highest RScores and AScores (from Table 1) being used to generate gene-gene interaction networks. Results showed that, for the RScore group, there were 441 connections among the 31 genes, which has significant literature support. In contrast, genes within the AScore group demonstrated only 15 relations among 19/31 genes (Fig. 3 (b)) with 12 genes showing no direct relations with other genes in the group (Fig. 3 (b); highlighted in green). This observation was consistent with the GSEA and SNEA, and suggests that genes with the lowest AScore were not as functionally close to each other as the RScore group.

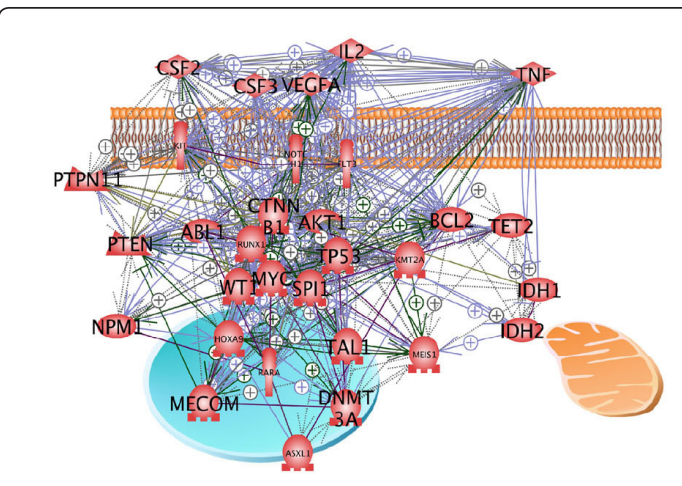

(a)

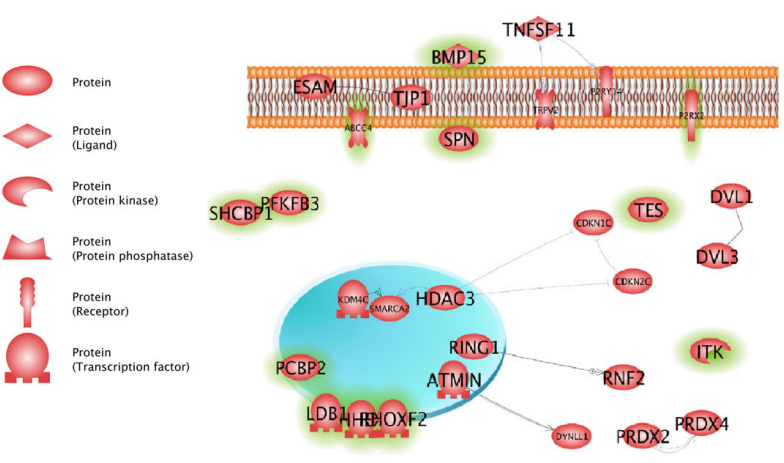

(b)

Fig. 3 Connectivity Networks built by 31 Genes from Different Groups. (a) 31 genes from RScore group; (b) 31 genes from AScore group. The networks were generated using Pathway Studio. Unrelated genes appear in green.

\subsection{EScore analysis}

Using GSEA, two biological metrics, EScore and PScore were generated for each gene. The PScore value represents how many leukemia associated pathways involved the gene. The EScore shows pathway significance.

A correlation analysis using averaged metric values of all 1,093 genes at a group level was conducted to compare the EScore and PScore with the two literature metrics (Fig. 4 (a)). A group size of 36 genes was used. The 1,093 genes were sorted by RScore, then averaged by each type of metrics values using a moving window of length 36 .

Results showed that the average scores strongly correlate, especially for the top ones. (Fig. 4 (a) and Table 6). Group-wise PScore and EScore were extremely correlated $(p=0.99)$.

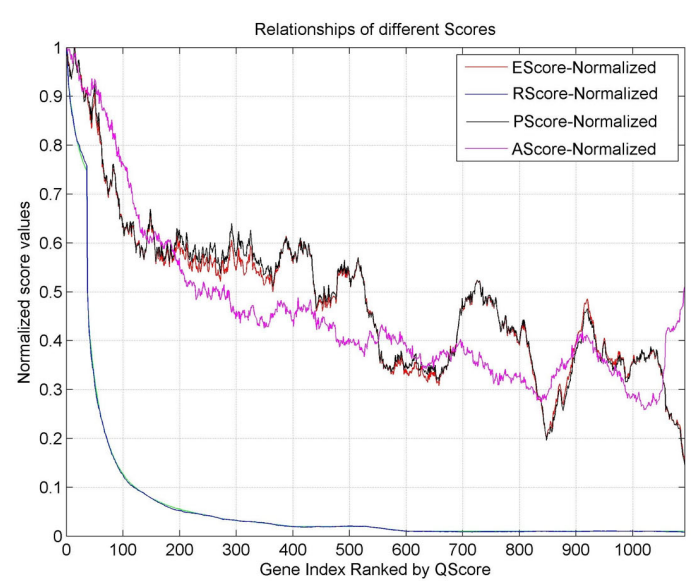

(a)

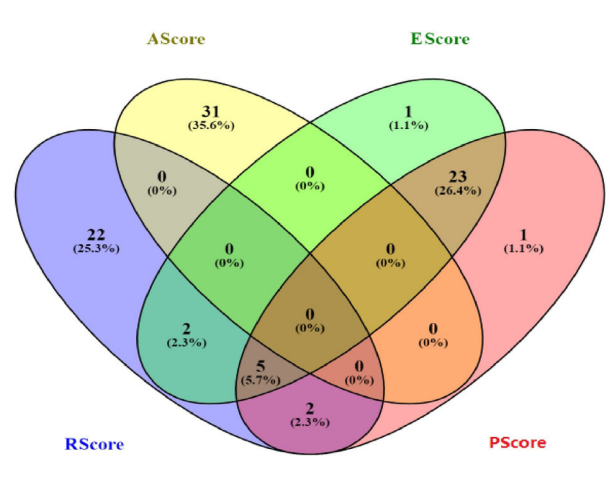

(b)

Fig. 4 Comparison of Different Metrics Ranking the 1,093 Genes. (a) Comparison of the average metrics values with gene set size of 36; (b) A Venn diagram of the top 31 genes selected by different metrics. 
Table 6. Pearson Correlation Coefficients between Different Metrics

\begin{tabular}{lcccc}
\hline & EScore & PScore & RScore & AScore \\
\hline RScore & 1.00 & & & \\
EScore & 0.99 & 1.00 & & \\
PScore & 0.72 & 0.74 & 1.00 & \\
AScore & 0.61 & 0.63 & 0.50 & 1.00 \\
\hline
\end{tabular}

In addition to the group-wise correlations analysis, a cross-analysis of the top 31 genes selected using different scores was performed and is presented in a Venn Diagram. (Fig.4 (b)) (Oliveros, 2007-2015).

There was a significant overlap between the PScore group and the EScore group (28/31). These 28 genes related to most pathways that were significantly enriched. Additionally, 5 genes were identified as being in overlapping EScore, PScore, and RScore groups, including TP53, CTNNB1, AKT1, TNF, and RARA (RScore: $58.80 \pm 11.17$ references, PScore: $34.00 \pm 2.55$ pathways) (Fig. 4 (b)). There were 23 genes observed in both the PScore group and the EScore group, but not in the RScore group. This included: RELA, JUN, EGFR, SRC, J AK2, HIF1A, TGFB1, HDAC1, STAT3, IL1B, PTK2, FYN, LCK, LYN, MAPK3, IL6, MAPK1, EP300, CREB1,
ERBB2, PDGFRB, GSK3B, and KDR. These genes played roles within multiple significant pathways with leukemia (32.04.28 pathways). Although they were older (AScore: 12.486.71 years) and were not frequently replicated (RScore: $10.04 \pm 8.94$ references), the results suggest that they are worthy of further study.

\subsection{Validation using expression data}

Significant leukemia candidate gene-gene sets were hypothesized as contributing to being able to distinguish leukemia patients from healthy controls. If the selected gene set $(1,093$ genes $)$ and the top genes selected by the proposed metric scores are significant to leukemia pathogenesis, then they should lead to significant higher classification accuracies when compared to randomly selected gene sets. To test this hypothesis that the 1,093-gene-pool and the 4 proposed metrics are effective, classification and leave-one-out (LOO) cross validation was conducted on a gene expression dataset (NCBI GEO: GSE50006). This was followed by a 5,000-run permutation test.

The 1,093 genes were ranked by different metric scores. The top $(=1,2, \ldots)$ genes were then used as input variables for classification and LOO cross validation. LOO results using different number of genes, with the maximum classification ratios (maxCRs) marked at the position of corresponding number of genes appear in Fig. 5 (See Table 7).

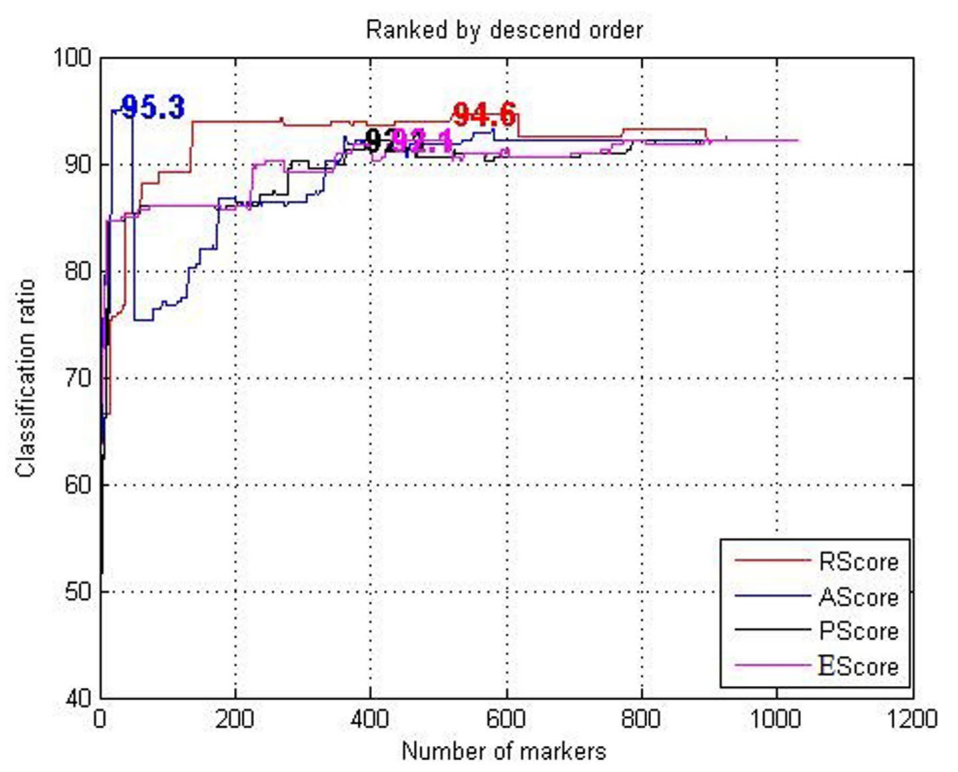

Fig. 5 Comparison of LOO Cross Validations Metrics. 
The top genes selected by different scores can lead to the highest classification accuracies, adding more variable/genes with lower score may not necessarily help, which demonstrates the effectiveness of the proposed metrics (Fig. 5). All four groups (RScore, AScore, PScore, and EScore), obtained the highest scores of $94.6 \%, 95.3 \%, 92.1$ $\%$ and $92.1 \%$, respectively, with a relatively small number of genes. All the permutation p-values of these groups passed the 0.05 threshold. The top 33 genes, by AScore, led to the highest CR (95.3\%), with a permutation $p$-value of 0.0054 . Employing all matched 1,031/1,093 genes, resulted in $92.1 \% \mathrm{CR}$ which was reached with a permutation $p$-value of 0.037 . This suggests that the majority of the 1,093 genes were effective for leukemia prediction. The results of LOO cross-validation and permutation approaches for different gene sets appear in Table 7.

Table 7. Permutation Test for Top Genes Corresponding to the Highest CRs

\begin{tabular}{llllll}
\hline & RScore & AScore & PScore & EScore & 1,031 Genes \\
\hline MaxCRs & 94.62 & 95.30 & 92.11 & 92.11 & 92.11 \\
\#Genes & 520 & 33 & 392 & 430 & 1031 \\
pvalue & 0.0084 & 0.0054 & 0.044 & 0.035 & 0.037 \\
\hline
\end{tabular}

\section{DISCUSSION}

This study proposed 4 network metrics to evaluate the 1,093 candidate genes within a genetic network for leukemia. It employed an independent gene expression data set to validate their efficiencies. GSEA, SNEA, and NCA were also used to study the pathogenic significance of these candidate genes in the disease.

The 1,093 genes identified were not equal in terms of publication frequency (RScore), novelties (AScore), or the functional diversity (EScore). Using the proposed quality metrics scores, the genes may be ranked according to different needs/ significance and the top ones selected for further analysis (see Supplementary Material 1). Some frequently replicated genes (with a high RScore) also demonstrate high EScore and PScore, such as TP53, CTNNB1, AKT1, TNF, and RARA (see Fig. 4 (b)). These genes have an average support of 58.80 \pm 11.17 references, and were connected to multiple, significantly-enriched, pathways $(34.00 \pm 2.55)$. The results suggest that these genes are biologically significant in the disease.

There were 23 genes observed in both the PScore group and the EScore group (Fig. 4 (b)) which were not in the RScore group. Although they were older (AScore: $12.48 \pm 6.71$ years) and were not frequently replicated (10.04 \pm 8.94 references), the results suggest that they merit further study.
One example, the gene RELA, regulation of NFkappa B transcription factor activity (0051092); aging (GO: 0016280); liver development (GO: 0001889); negative regulation of apoptotic process (GO: 0006916); positive regulation of cell proliferation (GO: 0008284); transcription factor complex (GO: 0005667); innate immune response (GO: 0002226); and, protein kinase binding (GO: 0019901) ${ }^{[17-26]}$. This suggests that these genes may play significant roles in leukemia pathology and, thus, merit additional study.

The results demonstrate that most genes identified in this study were included in previouslyimplicated leukemia pathways. This included 6 cell apoptosis pathways, 9 cell growth and proliferation pathways, 11 transcription factor pathways, 7 protein phosphorylation related pathways, 3 immune system pathways, 8 protein kinase related pathways, and 2 neuronal system pathways ${ }^{[21-27]}$. We hypothesize that the majority of these literature-reported genes, especially those identified from significantly enriched pathways, should be functionally linked to leukemia. Although there may be false positives from the separate studies in the publications, it is less likely that a numerous group of genes were falsely perturbed $^{[14]}$.

When members of a gene set exhibit strong cross-correlation, GSEA boosts the signal-to-noise 
ratio making it possible to detect modest changes in individual genes ${ }^{[14]}$. The NCA analysis showed that many of the frequently reported genes related to leukemia are functionally associated with one another (Fig. 3). This is supported by hundreds of scientific reports. It should be noted that 952/1,093 were included in the top 100 pathways enriched ( $p$-value $<3.3 e-020)$, and that $857 / 1,093$ in the top 20 pathways appear in Table 2 ( $p$-value < 3.7e-041) .

If "functionally related" is defined as coexistence within the same genetic pathway, then $87.1 \%$ of the 1,093 genes are functionally related. The results indicate that these functionally-linked genes are more likely to be true discoveries than noise (false positives). It is less likely that these functionally-related genes were falsely identified than a single gene.

A Sub-Network Enrichment Analysis (SNEA) was performed which provided high confidence levels when interpreting experimentally-derived genetic data against a background of previouslypublished results (Pathway Studio Web Help). SNEA results revealed that many of the 1,093 genes ( $>$ $90 \%$ ) have also been identified as causal genes for other health disorders such as, breast cancer, hepatocellular carcinoma, and lung cancer, all of which have strong associations with leukemia ${ }^{[28-30]}$.

A LOO cross-validation and permutation process using a gene expression data set (NCBI GEO: GSE50006) identified several significant gene combinations by using different scores, which generated the highest CRs. Permutation results showed that the top genes as determined by these four scores, as well as the 1,031/1,093 genes, were effective in predicting leukemia ( $p$-value $<0.05)$. This indicates the effectiveness of the proposed metric scores. The top 33 genes selected by AScore reached the highest $\mathrm{CR}, 95.3 \%$, with a permutation $p$-value of 0.0054 . This suggests that the genes identified in the earliest stage of leukemia genetic studies play a significant role in leukemia prediction.

This study has several limitations that should be considered in future work. The 1,093 genes were identified from leukemia-gene relation data extracted from the Pathway Studio ResNet database. Although supported by 6,524 articles, it is possible that some leukemia-gene relationships may have not been identified. The 4 proposed metrics were effective in selecting the top genes for leukemia prediction. Further network analysis with more experimental data may extract additional useful features for identifying biologically significant genes.

\section{CONCLUSION}

Leukemia is a complex, genetically-caused, disease with the genetic causes linked to a large gene network. Integrating network gene-disease relation data and experimental data, with GSEA, SNEA, and NCA, may provide and effective approach to identifying potential target genes. This study provides an overview map for the current field of genetic research of leukemia, which could be used as the basis in future biological/genetic studies.

\section{DECLARATION OF INTERESTS}

The authors declare no conflicts of interest.

\section{FUNDING}

This research received no specific grants from funding agencies in the public, commercial, or notfor-profit sectors.

\section{REFERENCES}

1. World Cancer Report 2014. World Health Organization Press. 2014.

2. Hutter JJ. "Childhood leukemia". Pediatrics rev/ Am Acad Pediatrics. 2010; 31(6): 234-241.

3. Dilara FA, Deniz AO, Mine M, Ustun E, Muhterem B, Emin K, Nejat A. Detection of TET2, KRAS and CBL variants by Next Generation Sequencing and analysis of their correlation with JAK2 and FLT3 in childhood AML. Egypt J Med Human Genet. 2016; 17(2): 209-215.

4. Zhang $R$, Yang J, Sun $H$, Jia $H$, Liao J, Shi $Y$, Li G. Comparison of minimal residual disease (MRD) monitoring byWT1 quantification between childhood acute myeloid leukemia and acute lymphoblastic leukemia. Eur Rev Med Pharmacol Sci. 2015; 19(14): 2679-2688.

5. Waring P, Tie J, Maru D, Karapetis C. RAS Mutations as predictive biomarkers in clinical management of metastatic colorectal cancer. Clin Colorect Cancer. 2015; 6(3): 314-321.

6. Jabbour E, Verstovsek S, Giles F, Varsha 
G, Cortes J, O'Brien S, Plunkett W, GarciaManero G, Jackson CE, Kantarjian H, Andreeff M. 2-Chlorodeoxyadenosine and cytarabine combination therapy for idiopathic hypereosinophilic syndrome. Cancer. 2005; 104(3): 541-546.

7. Rosseau RF, Ettore B, Dutour A. Immunotherapy of high-risk acute leukemia with a recipient (autologous) vaccine expressing transgenic human CD40L and IL-2 after chemotherapy and allogeneic stem cell transplantation. Blood. 2006; 107(4): 1332-1341.

8. Chen C, Armstrong S. Targeting DOT1L and HOX gene expression in MLL-rearranged leukemia and beyond. Exp Hematol. 2015; 43(8): 673-684.

9. Jelkmann W. Pitfalls in the measurement of circulating vascular endothelial growth factor. Clin Chem. 2001; 47(4): 617-623.

10. Duque-Afonso J, Feng J, Scherer F, Lin CH, Wong SH, Wang Z, Iwasaki M, Cleary ML. Comparative genomics reveals multistep pathogenesis of E2A-PBX1 acute lymphoblastic leukemia. J Clin Invest. 2015; 125(9): 36673680.

11. McCubrey JA, Steelman LS, Bertrand FE, Davis NM, Abrams SL, Montalto G, D'Assoro AB, Libra M, Nicoletti F, Maestro R, Basecke J, Cocco L, Cervello M, Martelli AM. Multifaceted roles of GSK-3 and Wnt/ $\beta$-catenin in hematopoiesis and leukemogenesis: opportunities fortherapeutic intervention. Leukemia. 2014; 28(1): 15-33.

12. Devine T, Dai M. Targeting the ubiquitin-mediated proteasome degradation of p53 for cancer therapy. Cur Pharm Design. 2013; 19(18): 32483262.

13. Laky K, Evans S, Perez -Diez A, Fowlkes B. Notch Signaling Regulates Antigen Sensitivity of Naive CD4+ T Cells by Tuning Co-stimulation. Immunity. 2015; 42(1): 80-94.

14. Subramanian A, Tamayo P, Mootha VK. Gene set enrichment analysis: a knowledgebased approach for interpreting genome-wide expression profiles. Proc Natl Acad Sci USA. 2005; 102(43): 15545-15550.

15. Sivachenko AY, Yuryev A, Daraselia N, Mazo I. Molecular networks in microarray analysis. J Bioinform Comput Biol. 2007; 5(2B): 429-456.

16. Wang J, Cao H, Liao Y, Liu W, Tan L, Tang Y, Chen J, Xu X, Li H, Luo C, Liu C, Merikangas KR. Three dysconnectivity patterns in treatmentresistant schizophrenia patients and their unaffected siblings. Neuroimage Clin. 2015; 8: 95-103.

17. Higashiyama S, Iwabuki $\mathrm{H}$, Morimoto $\mathrm{C}$, Hieda $\mathrm{M}$, Inoue H, Matsushita N. Membrane-anchored growth factors, the epidermal growth factor family: beyond receptor ligands. Cancer Sci. 2008; 99(2): 214-220.

18. Escárcega RO, Fuentes-Alexandro S, GarcíaCarrasco M, Gatica A, Zamora A. The transcription factor nuclear factor-kappa B and cancer. Clin Oncol (R Coll Radiol). 2007; 19(2): 154-161.

19. Lisanti MP, Martinez-Outschoorn UE, Lin Z, Sotgia F. Hydrogen peroxide fuels aging, inflammation, cancer metabolism and metastasis: the seed and soil also needs "fertilizer". Cell Cycle. 2011; 10(15): 2440-2449.

20. Hirabayashi K, Shiohara M, Takahashi D, Saito S, Tanaka M, Yanagisawa R, Sakashita K, Nakamura T, Ishii E, Koike K. Retrospective analysis of risk factors for development of liver dysfunction in transient leukemia of Down syndrome. Leuk Lymphoma. 2011; 52(8): 15231527.

21. Stahnke K, Eckhoff S, Mohr A, Meyer LH, Debatin KM. Apoptosis induction in peripheral leukemia cells by remission induction treatment in vivo: selective depletion and apoptosis in a CD34+ subpopulation of leukemia cells. Leukemia. 2003; 17(11): 2130-2139.

22. Clarkson B, Ohkita T, Ota K, Fried J. Studies of cellular proliferation in human leukemia. I. Estimation of growth rates of leukemic and normal hematopoietic cells in two adults with acute leukemia given single injections of tritiated thymidine. J Clin Invest. 1967; 46(4): 506-529.

23. Prange KH, Singh AA, Martens JH. The genomewide molecular signature of transcription factors in leukemia. ExpHematol. 2014; 42(8): 637-650.

24. Juan WC, Ong ST. The role of protein phosphorylation in therapy resistance and disease progression in chronic myelogenous leukemia. Prog Mol Biol Transl Sci. 2012; 106: 107-142.

25. Goldman D. Chronic lymphocytic leukemia and its impact on the immune system. Clin J Oncol Nurs. 2000; 4(5): 233-234.

26. Martínez-Cuadrón D, Montesinos P, PérezSirvent M, Avarla A, Cordon L, RodriguezVeiga R, Martin G, Sanz J, Martinez J, Sanz MA. Central nervous system involvement at first relapse in patients with acute myeloid leukemia. 
Haematologica. 2011; 96(9): 1375-1379.

27. Redig AJ, Platanias LC. Protein kinase C signalling in leukemia. Leuk Lymphoma. 2008; 49 (7): 1255-1262.

28. Valentini CG, Fianchi L, Voso MT, Pagano L. Incidence of Acute Myeloid Leukemia after Breast Cancer. Mediterr J Hematol Infect Dis. 2011; 3(1): e2011069.
29. Borgonovo G, Secondo V, Varaldo E, Pistoia V, Gobbi M, Mattioli FP. Large granular lymphocyte leukemia associated with hepatocellular carcinoma: a case report. Haematologica. 1996; 81(2): 172-174.

30. Libshitz HI, Zornoza J, McLarty JW. Lung cancer in chronic leukemia and lymphoma. Radiol. 1978; 127(2): 297-300. 